\title{
Aggregated CdS Quantum Dots: Host of Biomolecular Ligands
}

\author{
S. Shankara Narayanan and Samir Kumar Pal* \\ Unit for Nano Science \& Technology, Department of Chemical, Biological \& Macromolecular Sciences, \\ S. N. Bose National Centre for Basic Sciences, Block JD, Sector III, Salt Lake, Kolkata 700 098, India
}

Received: July 4, 2006; In Final Form: September 30, 2006

\begin{abstract}
In this contribution, we have studied structural and photophysical properties of aggregated CdS quantum dots (QDs) capped with 2-mercaptoethanol in aqueous medium. The hydrodynamic diameter of the nanostructures in aqueous solution was found to be $\sim 160 \mathrm{~nm}$ with the dynamic light scattering (DLS) technique, which is in close agreement with atomic force microscopy (AFM) studies (diameter $\sim 150 \mathrm{~nm}$ ). However, the UVvis absorption spectroscopy, powder X-ray diffraction (XRD), and transmission electron microscopy (TEM) studies confirm the average particle size (QD) in the nanoaggregate to be $4.0 \pm 0.5 \mathrm{~nm}$. The steady-state and time-resolved photoluminescence studies on the QDs further confirm preservation of electronic band structure of the QDs in the nanoaggregate. To study the nature of the nanoaggregate we have used small fluorescent probes, which are widely used as biomolecular ligands (2,6- $p$-toluidinonaphthalene sulfonate (TNS) and Oxazine 1 ), and found the pores of the aggregate to be hydrophobic in nature. The significantly large spectral overlap of the host quantum dots (donor) with that of the guest fluorescent probe Oxazine 1 (acceptor) allows us to carry out Förster resonance energy transfer (FRET) studies to estimate average donor-acceptor distance in the nanostructure, found to be $\sim 25 \AA$. The quantum dot aggregate and the characterization techniques reported here could have implications in the future application of the QD-nanoaggregate as host of small ligand molecules of biological interest.
\end{abstract}

\section{Introduction}

Over the past decades colloidal luminescent semiconductor nanocrystals, often referred to as "quantum dots" or "QDs", have been studied in detail due to their unique size-dependent optical and electronic properties. ${ }^{1,2}$ The increase in the band gap with the decrease in the size of the particles is the most identified aspect of quantum confinement in semiconductors. When the dimensions of nanocrystalline particles approach the exciton Bohr radius, a blue shift in energy is observed due to the quantum confinement phenomenon. The effective mass model is commonly used to study the size dependence of optical properties of QDs system. ${ }^{3}$ One of the typical features of nanoparticles is their spontaneous self-aggregation into functional structures driven by the energetics of the system, which are known as self-aggregated nanostructures. The aggregation through noncovalent interactions (e.g., hydrogen bonding interaction, $\pi-\pi$ interaction, charge-transfer interaction, acid/base proton transfer, van der Waals forces, host-guest interaction, and electrostatic forces) is an effective mechanism that has been proven successful in forming different nanoparticle assembly motifs such as 2D and 3D superlattices of nanocrystals., ${ }^{4,5}$

The study of aggregated molecular structures has recently attracted considerable attention due to their novel application as a host structure for small molecule guests. ${ }^{6}$ The host-guest chemistry is emerging as an important way to study how binding of molecules into space-restricted environments can mediate their chemical reactivity. ${ }^{7}$ The size, shape, and chemical environment of the aggregated host help in controlling the recognition and reactivity of guest molecules, offering the potential for entirely new types of synthetic catalysts. The

* Address correspondence to this author. E-mail: skpal@bose.res.in. Fax: 91-33-2335-3477. formation of confined and protected hydrophobic environment in the aggregated structure has played a vital role for the study of various hydrophobic ligands. One of the classical examples of crystalline molecules acting as host is zeolite. Various types of zeolites are characterized by unique uniform pores, and channels or cavities of various sizes. The unique arrangement of pores in them provides a three dimensionally restricted environment for the inclusion of various guest molecules. ${ }^{8}$

Here we report our studies on an aggregated nanostructure of CdS quantum dots (QDs) capped with 2-mercaptoethanol. By using steady state absorption/photoluminescence (PL) spectroscopy, Fourier transformed infrared spectroscopy (FTIR), powder X-ray diffraction (XRD), transmission electron microscopy (TEM), dynamic light scattering (DLS), and atomic force microscopy (AFM) we have characterized the nanoaggregation. The picosecond resolved PL measurements on the nanostructure reveal the electron-hole-pair recombination dynamics. The interactions of small biologically relevant fluorescent probes clearly demonstrate the efficacy of the nanoaggregate as a potential host for the small ligand molecules. We also estimated the average distance of QDs in the nanostructure from a chromophoric guest ligand by using steady state and picosecondresolved Förster resonance energy transfer (FRET).

\section{Materials and Methods}

Chemicals were obtained from the following sources: anhydrous $\mathrm{Na}_{2} \mathrm{~S}$ (Sigma Aldrich), $\mathrm{NaOH}$ (Merck), $\mathrm{Cd}\left(\mathrm{NO}_{3}\right)_{2} \cdot \mathrm{H}_{2} \mathrm{O}$ (Sigma Aldrich), 2-mercaptoethanol $\left(\mathrm{HSCH}_{2} \mathrm{CH}_{2} \mathrm{OH}\right)(\mathrm{SRL})$, 2,6- $p$-toluidinonaphthalene sulfonate (TNS) (Sigma-Aldrich), and Oxazine 1 (OX1; Exciton). The chemicals were of highest commercially available purity and were used as received. All aqueous solutions were prepared with deionized water from Millipore system and completely degassed by dry nitrogen. The 
preparation of 2-mercaptoethanol-capped CdS quantum dots (QDs) was carried out following the methodology described in the literature. ${ }^{9}$ Briefly, $2 \mathrm{~mL}$ of a $\mathrm{Na}_{2} \mathrm{~S}$ aqueous solution (10 $\mathrm{mM}$ ) was added dropwise to $100 \mathrm{~mL}$ of a $\mathrm{Cd}\left(\mathrm{NO}_{3}\right)_{2}$ aqueous solution $(0.2 \mathrm{mM})$ containing $1.4 \mu \mathrm{L}$ of 2-mercaptoethanol, under vigorous stirring. As mentioned in the literature, ${ }^{9}$ the final concentration of $\mathrm{CdS}$ in the aqueous solution is $0.2 \mathrm{mM}$. The $\mathrm{pH}$ of the final solution was adjusted to 10.5 by adding $\mathrm{NaOH}$ solution. The final colloidal solution was kept overnight in the dark under nitrogen atmosphere and all the studies were carried out the next day.

UV-vis absorption spectroscopy, steady-state photoluminescence (PL), powder X-ray diffraction (XRD), Fourier transform infrared spectroscopy (FTIR), dynamic light scattering (DLS), atomic force microscopy (AFM), and high-resolution transmission electron microscopy (HR-TEM) were done by Shimadzu Model UV-2450 spectrophotometer, Jobin Yvon Model Fluoromax-3 fluorimeter, PANalytical XPERT-PRO diffractometer equipped with $\mathrm{Cu} \mathrm{K} \alpha$ radiation $(\lambda=1.5418 \AA$ ) (at $40 \mathrm{~mA}, 40$ $\mathrm{kV}$ ), JASCO FT/IR-6300 spectrometer (transmission mode), Malvern instruments (model Nano-S), Veeco Instruments (CPII), and JEOL (JEM-2100), respectively. X-ray powder diffraction (XRD) patterns were obtained by employing a scanning rate of $0.02^{\circ} \mathrm{s}^{-1}$ in the $2 \theta$ range from $15^{\circ}$ to $75^{\circ}$. Samples for TEM were prepared by placing a drop of the colloidal solution on a carbon-coated copper grid and allowing the film to evaporate overnight at room temperature. To ensure that TEM images are of nanoparticles and not of dried salt $\left(\mathrm{NaNO}_{3}\right)$ remaining from the particle precipitation process, we dialyzed the aqueous solution of the QDs against water $(\mathrm{pH} 10.5)$ exhaustively using a dialysis membrane (Spectrum Laboratories, Inc, USA) with a molecular weight cutoff (MWCO) of 12 00014 000. The dialyzed solution was then placed on a separate carbon-coated copper grid and dried overnight at room temperature. The size of the nanoparticles was determined from the TEM images obtained at $200 \mathrm{kV}$. Sample for AFM was prepared by placing a drop of the colloidal solution on a silicon wafer and then allowing it to evaporate overnight at room temperature. For the FTIR measurements, powdered QD samples were mixed with $\mathrm{KBr}$ powder and pelletized. The background correction was made by using a reference blank of the $\mathrm{KBr}$ pellet.

DLS measurements employ a $4 \mathrm{~mW}$ He-Ne laser $(\lambda=632.8$ $\mathrm{nm})$ with a thermostatted sample chamber. All measurements are taken at $178^{\circ}$ scattering angle. The scattering intensity data are processed with the instrumental software to obtain the hydrodynamic diameter $\left(d_{\mathrm{H}}\right)$ and the size distribution of the scatterer in each sample. The instrument measures the timedependent fluctuation in intensity of light scattered from the particles in solution at a fixed scattering angle. Hydrodynamic diameters $\left(d_{\mathrm{H}}\right)$ of the particles are estimated from the intensity autocorrelation function of the time-dependent fluctuation in intensity. $d_{\mathrm{H}}$ is defined as

$$
\mathrm{d}_{\mathrm{H}}=k T / 3 \pi \eta D
$$

where $k=$ Boltzmann constant, $T=$ absolute temperature, $\eta=$ viscosity, and $D=$ translational diffusion coefficient. In a typical size distribution graph from the DLS measurement, the $X$-axis shows a distribution of size classes (in $\mathrm{nm}$ ), while the $Y$-axis shows the relative intensity of the scattered light. This is therefore known as an intensity distribution graph.

All photoluminescence transients were taken with use of the picosecond-resolved time-correlated single photon counting (TCSPC) technique. We used a commercially available pico- second diode laser-pumped time-resolved fluorescence spectrophotometer from Edinburgh Instruments, UK. To excite QD aggregate and $\mathrm{OX} 1$, picosecond excitation pulses from diode lasers with excitation wavelengths 375 and $633 \mathrm{~nm}$ respectively were used (instrument response function $($ IRF $)=80 \mathrm{ps}$ ). TNS molecules were excited by a pulsed light emitting diode (LED) at $299 \mathrm{~nm}(\mathrm{IRF}=450 \mathrm{ps})$. The photoluminescence from the sample was detected by a microchannel-plate-photomultiplier tube (MCP-PMT) after dispersion through a monochromator. For all transients the polarizer on the emission side was adjusted to be at $55^{\circ}$ (Magic angle) with respect to the polarization axis of the excitation beam. The observed fluorescence transients were fitted by using a nonlinear least-squares fitting procedure to a function $\left(X(t)=\int_{0}^{\mathrm{t}} E\left(t^{\prime}\right) R\left(t-t^{\prime}\right) \mathrm{d} t^{\prime}\right)$ comprised of convolution of the IRF $(E(t))$ with a sum of exponentials $(R(t)$ $\left.=A+\sum_{i=1}^{N} B_{i} \mathrm{e}^{-t / \tau_{i}}\right)$ with preexponential factors $\left(B_{i}\right)$, characteristic lifetimes $\left(\tau_{i}\right)$, and a background $(A)$. Relative concentration in a multiexponential decay was finally expressed as

$$
c_{n}=\left(B_{n} \tau_{n}\right) /\left(\sum_{i=1}^{N} B_{i} \tau_{i}\right) \times 100
$$

To estimate the fluorescence resonance energy transfer efficiency of the donor (CdS-QD) and hence to determine the distance of the donor-acceptor pair, we followed the methodology described in chapter 13 of ref 10 . The Förster distance $\left(R_{\mathrm{o}}\right)$ is given by

$$
R_{\mathrm{o}}=0.211\left[\kappa^{2} n^{-4} Q_{\mathrm{D}} J(\lambda)\right]^{1 / 6} \text { (in } \AA \text { ) }
$$

where $\kappa^{2}$ is a factor describing the relative orientation in space of the transition dipoles of the donor and acceptor. For donor and acceptors that randomize by rotational diffusion prior to energy transfer, the magnitude of $\kappa^{2}$ is assumed to be $2 / 3$. In the present study the same assumption has been made. The refractive index $(n)$ of the medium is assumed to be 1.4. $Q_{\mathrm{D}}$, the quantum yield of the donor QD in the absence of acceptor, is measured to be 0.016 . $J(\lambda)$, the overlap integral, which expresses the degree of spectral overlap between the donor emission and the acceptor absorption, is given by

$$
J(\lambda)=\frac{\int_{0}^{\infty} F_{\mathrm{D}}(\lambda) \epsilon(\lambda) \lambda^{4} \mathrm{~d} \lambda}{\int_{0}^{\infty} F_{\mathrm{D}}(\lambda) \mathrm{d} \lambda}
$$

where $F_{\mathrm{D}}(\lambda)$ is the fluorescence intensity of the donor in the wavelength range of $\lambda$ to $\lambda+\mathrm{d} \lambda$ and is dimensionless. $\epsilon(\lambda)$ is the extinction coefficient (in $\mathrm{M}^{-1} \mathrm{~cm}^{-1}$ ) of the acceptor at $\lambda$. If $\lambda$ is in $\mathrm{nm}$, then $J(\lambda)$ is in units of $\mathrm{M}^{-1} \mathrm{~cm}^{-1} \mathrm{~nm}^{4}$. Once the value of $R_{\mathrm{o}}$ is known, the donor-acceptor distance $(r)$ can easily be calculated by using the formula

$$
r^{6}=\left[R_{\mathrm{o}}^{6}(1-E)\right] / E
$$

Here $E$ is the efficiency of energy transfer. The transfer efficiency is measured by using the relative fluorescence intensity of the donor in the absence $\left(F_{\mathrm{D}}\right)$ and presence $\left(F_{\mathrm{DA}}\right)$ of the acceptor. The efficiency $E$ is also calculated from the lifetimes under these respective conditions ( $\tau_{\mathrm{D}}$ and $\left.\tau_{\mathrm{DA}}\right)$.

$$
\begin{aligned}
& E=1-\left(F_{\mathrm{DA}} / F_{\mathrm{D}}\right) \\
& E=1-\left(\tau_{\mathrm{DA}} / \tau_{\mathrm{D}}\right)
\end{aligned}
$$




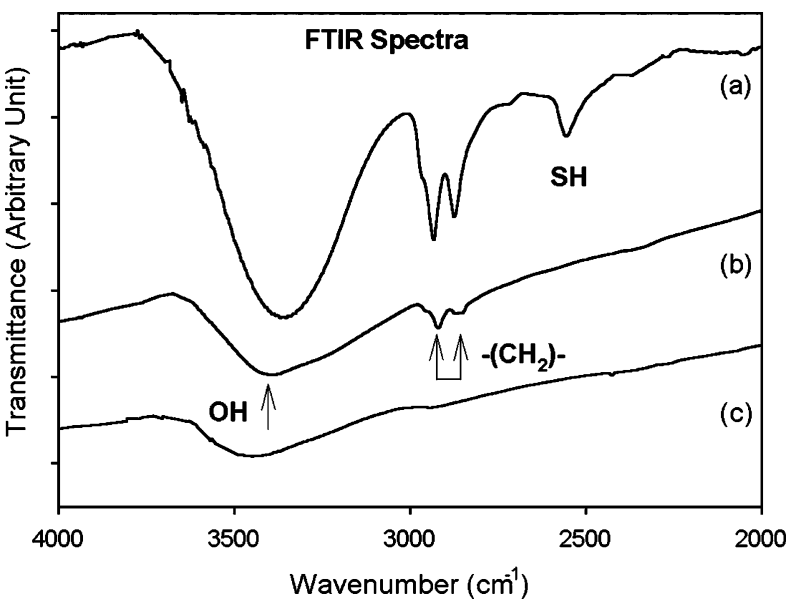

Figure 1. Fourier transform infrared (FTIR) spectra: (a) 2-mercaptoethanol, (b) 2-mercaptoethanol-capped CdS QDs, and (c) CdS QDs without any capping agent.

The distances measured with eqs $5 \mathrm{a}$ and $5 \mathrm{~b}$ are revealed as $R^{\mathrm{S}}$ (steady state measurement) and $R^{\mathrm{TR}}$ (measurement), respectively.

\section{Results and Discussion}

The FTIR transmittance spectrum for the CdS nanoparticles (Figure 1) capped with 2-mercaptoethanol is compared with those of pure 2-mercaptoethanol and $\mathrm{CdS}$ nanoparticles prepared without the capping agent. Figure 1a clearly reveals the $\mathrm{S}-\mathrm{H}$ stretching vibration band of 2-mercaptoethanol at $2555 \mathrm{~cm}^{-1}$. A broadband around $3400 \mathrm{~cm}^{-1}$ and a strong doublet at 2873 and $2933 \mathrm{~cm}^{-1}$ in the spectrum of 2-mercaptoethanol are assigned to the $\mathrm{O}-\mathrm{H}$ stretching vibration and the symmetric and asymmetric stretching vibrations of the $\mathrm{CH}_{2}$ groups, respectively. ${ }^{11}$ On the other hand, there is no peak in the same region between 2000 and $3000 \mathrm{~cm}^{-1}$ in the spectrum of the $\mathrm{CdS}$ nanoparticles prepared in the absence of 2-mercaptoethanol as evident from Figure 1c. Whereas the peak due to the $\mathrm{CH}_{2}$ groups of 2-mercaptoethanol is also observed for the $\mathrm{CdS}$ nanoparticles capped with 2-mercaptoethanol (Figure 1b), the absorption of the $\mathrm{S}-\mathrm{H}$ stretching vibration completely disappeared after the capping. The observation clearly excludes that 2-mercaptoethanol simply coexists with the CdS nanoparticles. Since the surface capping of sulfide particles by thiols is attributed to coordination of the deprotonated mercapto groups to the sulfide surfaces, these IR spectra strongly confirm the surface capping of the CdS nanoparticles by direct bonding of the deprotonated mercapto group of 2-mercaptoethanol, presumably to the cadmium site at the surface. These results also suggest that the primary hydroxyl groups at the other end of the capping thiol can serve as a surface hydrophilic moiety of the capped $\mathrm{CdS}$ nanoparticles, and $\mathrm{H}_{2} \mathrm{O}$ might be adsorbed more readily on the modified sulfide surfaces. The UV-vis absorption spectrum of CdS QDs shown in Figure 2a exhibits absorption edge that is blue-shifted with respect to the bulk CdS ( $\sim 515$ $\mathrm{nm}$ ), arising from quantum confinement effect in the nanoparticles. ${ }^{12,13}$ An absorption band around $370 \mathrm{~nm}$ is observed, which indicates a relatively narrow size distribution of $\mathrm{CdS}$ nanoparticles. From the position of the absorption edge, the average particle size can be determined by using the well-established relation between particle size and absorption onset. ${ }^{14,15}$ The absorption edge $\left(\lambda_{\mathrm{e}}\right)$ is converted into the corresponding particle size by using Henglein's empirical curve that relates the absorption edge $\left(\lambda_{\mathrm{e}}\right)$ to the diameter $(2 R)$ of the particles.

$$
2 R_{\mathrm{CdS}}=0.1 /\left(0.1338-0.0002345 \lambda_{\mathrm{e}}\right) \mathrm{nm}
$$
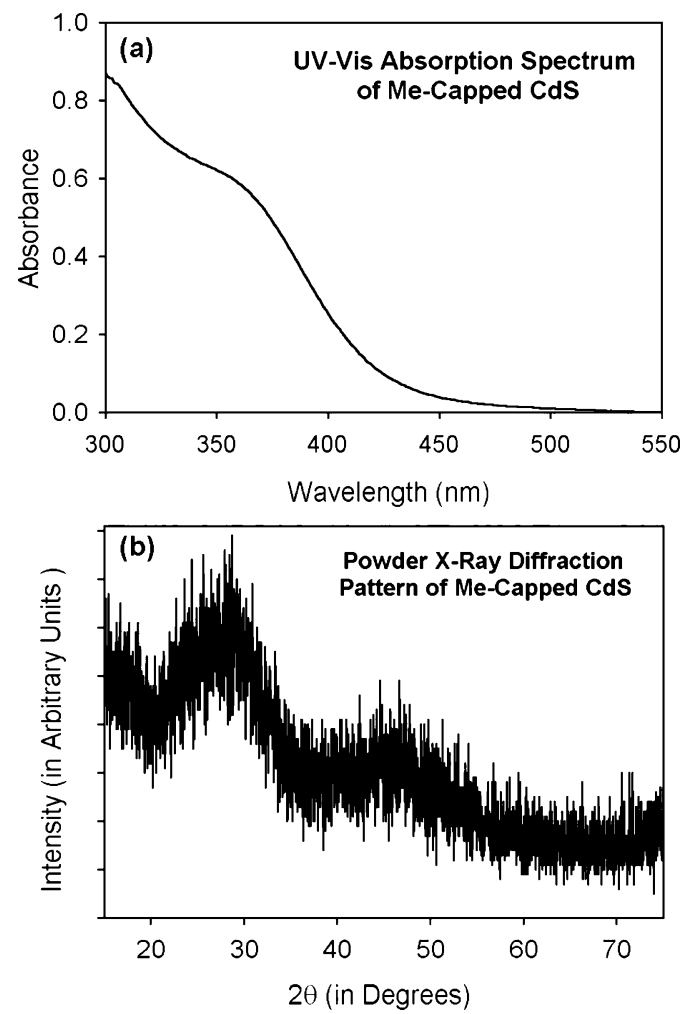

Figure 2. (a) UV-vis absorption spectrum of 2-mercaptoethanolcapped CdS-QDs. (b) Powder X-ray diffraction (XRD) pattern of 2-mercaptoethanol-capped CdS.

From Figure $2 \mathrm{a}$, the absorption edge $\left(\lambda_{\mathrm{e}}\right)$ is obtained by the intersection of the sharply decreasing region of the spectrum with the baseline ${ }^{14}$ and found to be $429.5 \mathrm{~nm}$. Thus from eq 6 the particle size was estimated to be $3 \mathrm{~nm}$.

To estimate the band gap energy, the effective mass model is used. ${ }^{3}$ The blue shift of band gap energy with the decrease in the diameter of QDs is described by the following equation

$$
E_{\mathrm{g}}^{\mathrm{eff}}=\mathrm{E}_{\mathrm{g}}+\hbar^{2} \pi^{2} / 2 \mu \mathrm{R}^{2}
$$

where $R$ is the particle radius, $\mu$ is the effective reduced mass, $E_{\mathrm{g}}$ is the bulk band gap energy $(2.5 \mathrm{eV}), E_{\mathrm{g}}^{\mathrm{eff}}$ is the effective band gap energy, and $\hbar=h / 2 \pi, h$ being Planck's constant. As the effective mass of the electrons is much smaller than that of the holes $\left(m_{\mathrm{e}}^{*}=0.2, m_{\mathrm{h}} *=0.8\right)$, the charge carrier confinement mainly affects the energetic level of the electrons. ${ }^{16}$ By using the estimated particle size from absorption edge, the effective band gap of CdS QDs is found to be $2.76 \mathrm{eV}$, which agrees well with the confinement regime. The powder XRD pattern for CdS nanocrystals is shown in Figure $2 b$. The XRD pattern apparently exhibits only two broad peaks, centered at $2 \theta \approx 28^{\circ}$ and $47^{\circ}$. However, a more careful study of the pattern suggests that these two peaks are overlaps of multiple peaks. This is also indicated by the marked asymmetry of the peak centered at $28^{\circ}$, resulting from the overlap of the reflection peaks (100), (002), and (101) of the hexagonal (Wurtzite) structure. The diffraction pattern is consistent with that of hexagonal CdS phase. The XRD patterns are considerably broadened due to the quantum size effect of nanocrystallites. ${ }^{17}$ The mean particle size was calculated by Debye-Scherrer's formula:

$$
D=0.9 \lambda / \beta \cos \theta
$$

where $D$ is the diameter of the nanocluster, $\lambda$ the wavelength of the incident $\mathrm{X}$-rays, $\beta$ is the full-width at the half-maximum, 

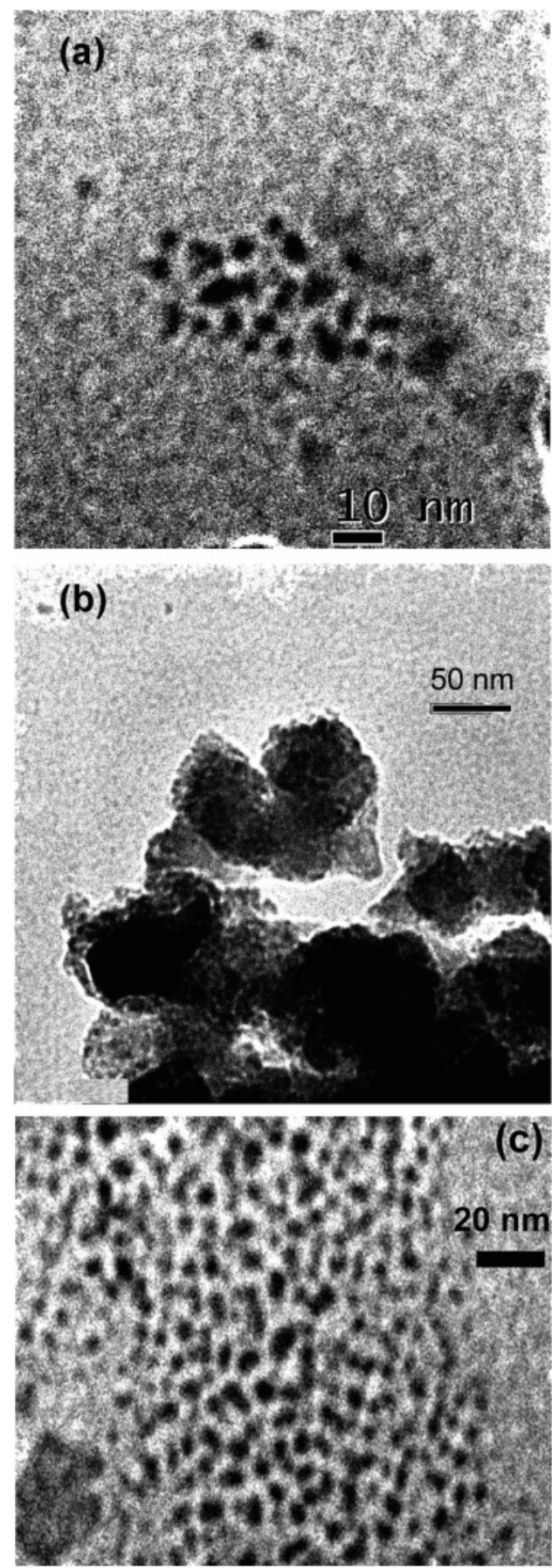

Figure 3. Transmission electron micrographs (TEM) of undialyzed sample: (a) individual QDs (100K magnification) and (b) nanoassembly (60K magnification). (c) Individual QDs in a dialyzed sample (60K magnification).

and $\theta$ is the diffraction angle. The average crystal size of the CdS QDs estimated by using Debye-Scherrer formula is 4.6 $\mathrm{nm},{ }^{18}$ which is slightly larger than that calculated by UV-vis absorption edge. The TEM image at $100 \mathrm{~K}$ magnification (Figure 3a) confirms the average particle diameter of the QDs to be 4.2 $\pm 0.5 \mathrm{~nm}$, which is similar to that obtained by XRD measurement. The particle size and distribution from our TEM studies are consistent with that of the reported value of $4.0 \pm 0.6 \mathrm{~nm} .{ }^{9}$ However, from the TEM experiments it is also found that most of the QDs were aggregated to a larger structure of average diameter $\sim 150 \mathrm{~nm}$ (Figure $3 \mathrm{~b}$ ). The TEM image of the dialyzed QDs is shown in Figure 3c. At $60 \mathrm{~K}$ and $120 \mathrm{~K}$ magnification (200 KV) the average particle size of the QDs is confirmed to
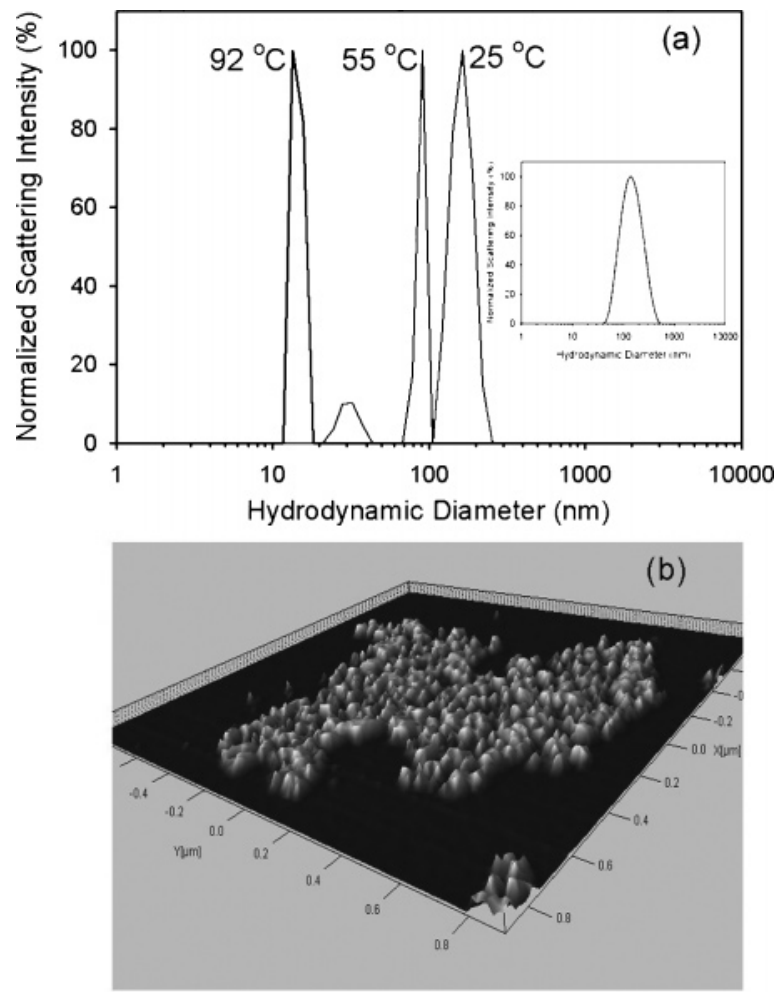

Figure 4. (a) Intensity-size distribution graph from dynamic light scattering (DLS) of dialyzed CdS QD in aqueous solution at three different temperatures. The inset shows size distribution of the QDaggregate in an undialyzed sample at $25{ }^{\circ} \mathrm{C}$. (b) Atomic force microscopy (AFM) image of the QD film on a silicon wafer.

be $4.5 \pm 0.5 \mathrm{~nm}$. In the micrograph of the dialyzed sample, the average diameter of the aggregated nanoassembly is found to be somewhat smaller $(\sim 50 \mathrm{~nm})$ than that of the samples without dialysis (Figure $3 b$ ). The reduction of aggregation size in the dialyzed sample may be due to the absence of excess salts in the solution, which were removed during dialysis. Our TEM experiments clearly confirm that the nanoparticles in the micrograph are free from excess salt clusters. The intensity distribution graph of the DLS experiment on the undialyzed CdS-QD solution as shown in the inset of Figure 4a indicates that the QDs were aggregated to form a nanostructure of average diameter $\sim 160 \mathrm{~nm}$ at $25^{\circ} \mathrm{C}$ with a broad distribution width of $191 \mathrm{~nm}$. As shown in Figure 4a the average diameter and width of QD-aggregate in the dialyzed solution are 175 and $25 \mathrm{~nm}$, respectively. The observation indicates the effect of excess ions of the undialyzed QD solutions on the size and distribution of the nanoaggregates. To investigate the nature of aggregation of the QDs, we performed temperature-dependent DLS studies on the dialyzed QD aqueous solutions. As shown in Figure $4 \mathrm{a}$ and Table 1 the aggregate size decreases (from $185 \mathrm{~nm}$ at $25^{\circ} \mathrm{C}$ to $18 \mathrm{~nm}$ at $92^{\circ} \mathrm{C}$ ) upon increase in temperature. The width of the size distribution also decreases (from $70 \mathrm{~nm}$ at $25^{\circ} \mathrm{C}$ to 12 $\mathrm{nm}$ at $92{ }^{\circ} \mathrm{C}$ ) with the increase in the temperature of the solution indicating essentially monodispersed size distribution at higher temperature. We also observed that the size of the QD aggregate almost resembles its original value again at $25^{\circ} \mathrm{C}(165 \mathrm{~nm})$. The observation clearly indicates that the aggregation is essentially driven by noncovalent interactions between QDs, which is expected to arise from the $(\mathrm{OH}-\mathrm{OH})$ hydrogen bonding of the exposed hydrophilic hydroxyl groups. The flocculated nanoaggregate is also evident from AFM (Figure $4 b)$ experiments. The average diameter of the nanoaggregate from the AFM experiment is found to be $150 \pm 5 \mathrm{~nm}$. An 

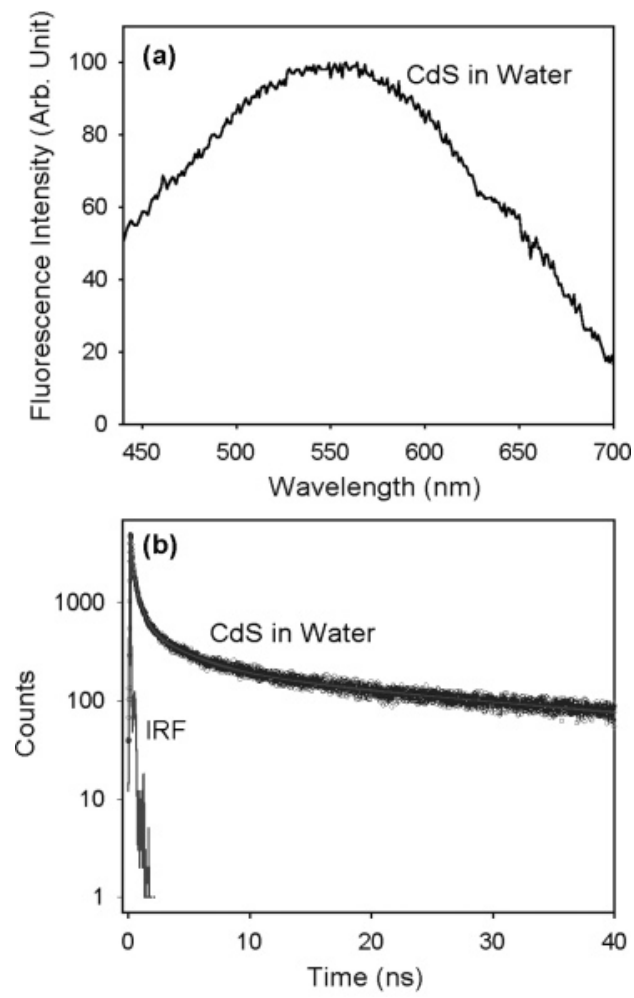

Figure 5. (a) Photoluminescence (PL) spectrum of CdS QDs in water. (b) Picosecond-resolved PL transients of CdS QDs in water.

TABLE 1: Temperature-Dependent Dynamic Light Scattering (DLS) Data of the Dialyzed Aqueous Solution of Thiol-Stabilized CdS-QDs

\begin{tabular}{ccccc}
\hline \multicolumn{2}{c}{ increasing temp } & & \multicolumn{2}{c}{ decreasing temp } \\
\cline { 5 - 6 } $\begin{array}{cccc}\text { temp } \\
\left({ }^{\circ} \mathrm{C}\right)\end{array}$ & $\begin{array}{r}\text { av aggregate } \\
\text { size }(\mathrm{nm})\end{array}$ & & $\begin{array}{c}\text { temp } \\
\left({ }^{\circ} \mathrm{C}\right)\end{array}$ & $\begin{array}{c}\text { av aggregate } \\
\text { size }(\mathrm{nm})\end{array}$ \\
\hline 25 & 185 & & 92 & 18 \\
35 & 112 & & 85 & 89 \\
45 & 104 & & 75 & 105 \\
55 & 89 & & 65 & 109 \\
65 & 68 & & 55 & 110 \\
75 & 68 & & 45 & 122 \\
85 & 58 & 35 & 136 \\
92 & 18 & 25 & 163
\end{tabular}

extensive structural characterization along with functionality of the aggregate of thiol-stabilized QDs will be left for future investigation.

Figure 5a shows the emission spectrum of CdS-QDs in aqueous solution excited at $350 \mathrm{~nm}$, which displays a broad emission band from 450 to $700 \mathrm{~nm}$. To examine the effect of excitation wavelengths on the observed photoluminescence from the QDs we used excitation from 300 to $375 \mathrm{~nm}$ and found insignificant change in the PL spectra. Reasonably small excitation wavelength dependency of the emission spectra confirms narrow dispersion of the $\mathrm{CdS}$ size in the nanostructure, ${ }^{19}$ which is in agreement with the TEM studies on the nanoassembly. ${ }^{9}$ To understand the dynamics of excited electronhole-pair (EHP) recombination, we measured picosecondresolved PL of the QDs. Figure 5b shows picosecond-resolved transient of CdS QDs (laser excitation $=375 \mathrm{~nm}$, emission wavelength $=500 \mathrm{~nm}$ ), which is found to be multiexponential in nature with three decay time components $\left(\tau_{i}\right)$ with various preexponential values $\left(a_{i}\right) ; 172 \mathrm{ps}(83 \%), 1.61 \mathrm{~ns}(13 \%)$, and $21.95 \mathrm{~ns}(4 \%)$. The overall PL decay leads to an average decay time $\left\langle\tau_{\mathrm{av}}\right\rangle=a_{1} \tau_{1}+a_{2} \tau_{2}+a_{3} \tau_{3}=1.23 \mathrm{~ns}$. Although a detail physical or mathematical model of the carrier recombination
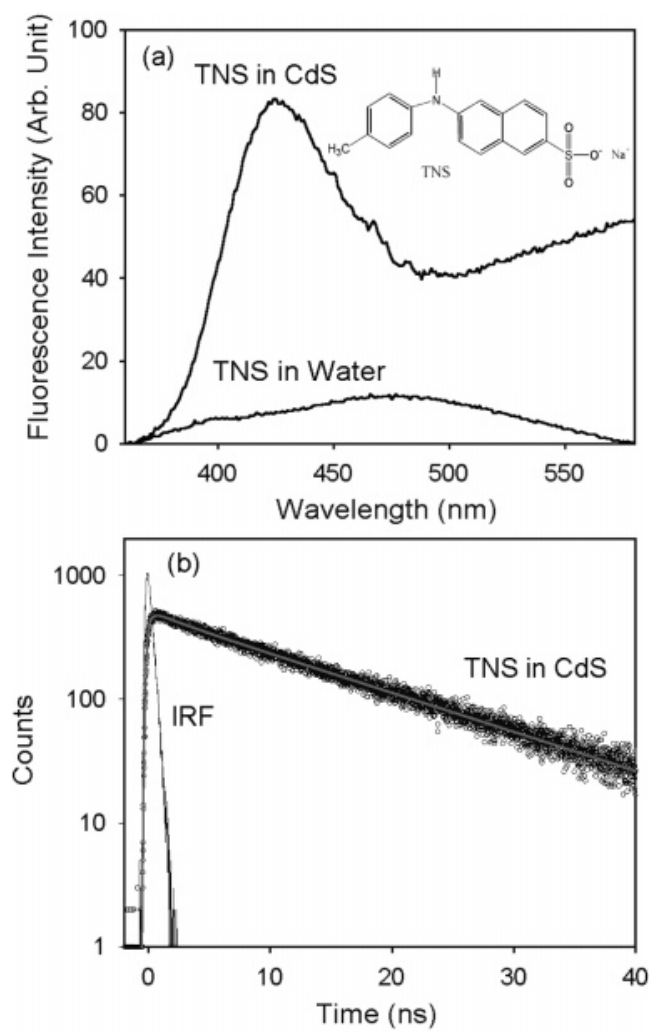

Figure 6. (a) Steady-state fluorescence spectrum of TNS in the presence of CdS QDs. The spectrum of TNS in bulk water is also shown for comparison. The inset shows the chemical structure of TNS. (b) Picosecond-resolved fluorescence transient of TNS encapsulated in the nanoassembly.

dynamics of II-VI semiconductor nanocrystals upon photoexcitation is lacking in the present literature, ${ }^{20,21}$ a recent attempt from our group has been made ${ }^{22}$ to rationalize picosecond dynamics of PL-decay of $\mathrm{CdS}$ nanoparticles suspended in reverse micellar solution. Relatively faster dynamics (172 ps and $1.61 \mathrm{~ns}$ ) in the PL-decay have been discussed ${ }^{22}$ in the context of the controversial "phonon bottleneck" effect. ${ }^{21}$ As pointed out in the literature, ${ }^{20,23}$ another possibility of the faster PL-decay dynamics is nonradiative recombination to the underlying trap states.

To study the efficacy of the nanostructure in hosting small organic ligands, we used a fluorescent biomolecular probe TNS in the aqueous solution of QDs. The anionic TNS, one of the most popular biological probes, ${ }^{24,25}$ binds mainly with the positively charged basic residues of a protein by noncovalent electrostatic interactions; there is also a possibility for TNS to bind with hydrophobic domain. ${ }^{26-28}$ The chemical structure of the probe TNS is shown in the inset of Figure 6a. To ensure maximum complexation of the TNS with the QDs, the concentration of the probe is maintained to be much lower $(0.05 \mathrm{mM})$ than that of the QDs $(0.2 \mathrm{mM})$ in the solution. The nanostructures of mercaptoethanol capped $\mathrm{CdS}$ particles have a $-\mathrm{CH}_{2}-$ $\mathrm{CH}_{2}-$ group in close vicinity of the surface of the QDs. Thus the immediate environments of the QDs are expected to be hydrophobic in nature due to presence of the small alkyl chain $\left(-\mathrm{CH}_{2}-\mathrm{CH}_{2}-\right)$ and can act as a possible host of the hydrophobic ligand TNS. However, the overall hydrophilic (water soluble) nature of the QDs comes from the exposure of hydroxyl groups to the bulk water. Figure 6a presents the emission spectra of TNS (excitation at $300 \mathrm{~nm}$ ) in aqueous solution in the presence and the absence of the CdS QD assembly. The emission peak $(470 \mathrm{~nm})$ of TNS in water is found to be 

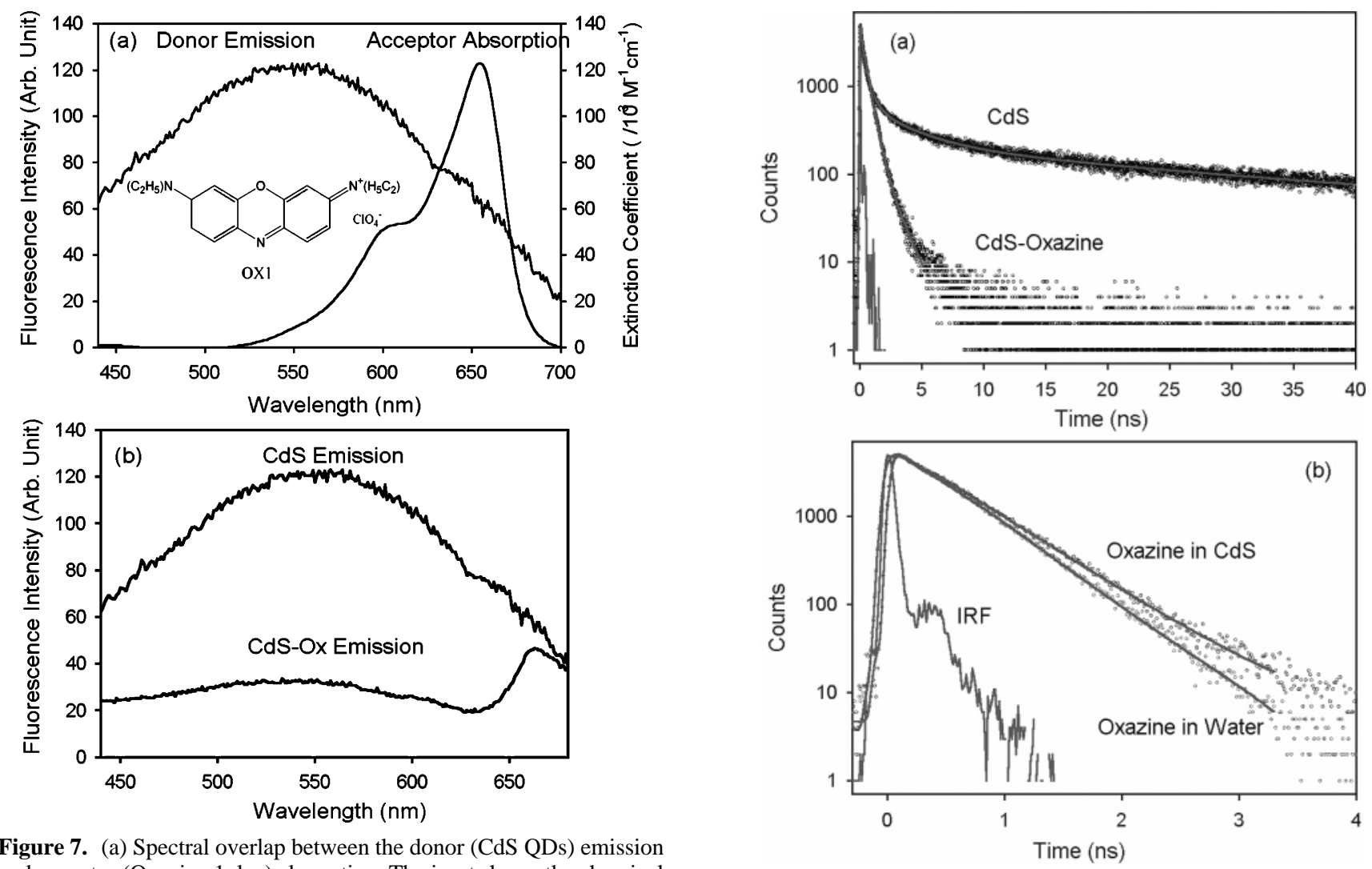

Figure 7. (a) Spectral overlap between the donor (CdS QDs) emission and acceptor (Oxazine 1 dye) absorption. The inset shows the chemical structure of OX1. (b) Steady-state photoluminescence quenching CdS QDs (donor) in the presence of acceptor oxazine 1 dye.

significantly blue-shifted in the presence of QD assembly (emission peak $=427 \mathrm{~nm}$ ). The fluorescence decay of the TNS probe in the presence of the nanoassembly appeared to be (Figure 6b) several orders of magnitude longer (50 ns) than that in bulk water (75 ps). ${ }^{29}$ The blue shift, significant intensity increase in the emission spectrum, and the lengthening of fluorescence lifetime of TNS in the presence of the nanoassembly clearly indicate geometrical restriction (caging) of the probe in the nanostructure. As detailed in an early study ${ }^{29}$ the major excited-state deactivation pathway of TNS is twisted intramolecular charge transfer (TICT). We also checked that free $\mathrm{Cd}^{2+}$ and $\mathrm{S}^{2-}$ ions have no effect on TNS emission. In a nonpolar solvent, the energy of charge transfer (CT) moves up because of the lack of stabilization by the solvent molecules. The lifetime of the probe TNS in an aprotic, nonpolar solvent was reported to be $7.3 \mathrm{~ns}$ with a maximum emission at $420 \mathrm{~nm}$ and a quantum yield of $0.3 .^{30}$

We also found that a cationic dye (Oxazine 1; OX1), which is commonly used to investigate the electron-transfer dynamics in DNA, ${ }^{31}$ can also be incorporated in the nanoaggregate. The chemical structure of OX1 is shown in the inset of Figure 7a. The efficiency of the nanoassembly to host anionic (TNS) and cationic (OX1) reflects the hydrophobic nature of the pores of the nanostructure (nanopores). In Figure 7a, the spectral overlap of the donor (CdS QDs) emission and absorption of OX1 (energy acceptor) is shown. It is noted that the absorption maximum of the acceptor is $650 \mathrm{~nm}$, which overlaps appreciably with the tail of the emission band of the CdS QDs. This favors the energy transfer from CdS QDs to OX1 dye. Thus the incorporation of $\mathrm{OX} 1$ into the nanopores gives us opportunity to estimate the average distance of the QDs from the encapsulated ligand molecules (e.g., OX1). As shown in Figure 7b the overall intensity of the CdS QDs emission drastically decreases

Figure 8. (a) Picosecond-resolved fluorescence transients of CdS QDs and the QD-nanoaggregate containing oxazine 1 dye in aqueous solution. (b) Picosecond-resolved fluorescence transients of oxazine1 in the absence and the presence of CdS QDs in aqueous solution.

upon incorporation of OX1, confirming the energy transfer from CdS QDs to the dye molecule. We also checked that free $\mathrm{Cd}^{2+}$ and $\mathrm{S}^{2-}$ ions have no effect on OX1 emission.

Figure 8a shows picosecond-resolved transients of pure CdS QDs and CdS QDs containing OX1. It has to be noted that OX1 was incorporated after the formation of QDS. To reduce the amount of free OX1 in the bulk water, the concentration of OX1 is maintained to be lower $(0.1 \mathrm{mM})$ than that of the QDs $(0.2$ $\mathrm{mM})$ in the solution. The transient of CdS QDs in the presence of the dye (Excitation at $375 \mathrm{~nm}$, Emission at $500 \mathrm{~nm}$ ) is also multiexponential with three decay components of $\left(\tau_{1}\right) 52$ ps $(52 \%),\left(\tau_{2}\right) 424$ ps $(40 \%)$, and $\left(\tau_{3}\right) 985$ ps $(8 \%)$ leading to an average decay time $\left\langle\tau_{\mathrm{av}}\right\rangle=a_{1} \tau_{1}+a_{2} \tau_{2}+a_{3} \tau_{3}=0.27 \mathrm{~ns}$. The significant drop of the average PL decay time compared to that of the nanostructure without OX1 (1.23 ns) also confirms the energy transfer occurs from CdS QDs to OX1 due to dipolar coupling. Although theoretical calculations to predict dipolar emission of a QD with wurtzite crystal structure have been advanced, ${ }^{32}$ very few experimental results ${ }^{33}$ have been published on the existence of excited-state dipole moment in semiconductor quantum dots. In a recent study from our group ${ }^{22}$ on $\mathrm{CdS}$ nanoparticles in reverse micellar solution at $300 \mathrm{~K}$, we reported physical motion of the dipolar QDs (photoluminescence anisotropy) having different shapes.

The calculated (from eqs 5a and 5b) donor to acceptor energy transfer efficiency from steady state and time-resolved studies is $74.3 \%$ and $77.64 \%$, respectively. The estimated donoracceptor distances from steady state and time-resolved experiments are 26.5 and $25.7 \AA$, respectively. The donor-acceptor distance of $\sim 2.5 \mathrm{~nm}$ is similar to the radius of the QDs, which is consistent with the fact that the acceptor OX1 resides at the surface sites of the QDs to quench the radiation of the donor 
dipole at the center of the QD. It is to be noted that quenching of PL intensity and shortening of the excited-state lifetime of the QDs (Figures 7 and 8) are also the signature of electron transfer to the guest OX1 as both the donor and acceptor are well-known to take part in excited-state electron-transfer reaction. ${ }^{8,34}$ In the case of electron transfer to/from OX1 in the nanoaggregate the excited-state lifetime of the dye is expected to be altered compared to that in bulk aqueous environments. ${ }^{34}$ As shown in Figure $8 \mathrm{~b}$ the fluorescence transient (excitation $633 \mathrm{~nm}$, emission $690 \mathrm{~nm})$ of OX1 in aqueous solution $\left(\left(\tau_{1}\right)\right.$ 37.5 ps $(1.15 \%),\left(\tau_{2}\right) 411$ ps $(94.65 \%)$, and $\left(\tau_{3}\right) 1.26$ ns $\left.(4.2 \%)\right)$ is found to be similar to that in the CdS nanoaggregate $\left(\left(\tau_{1}\right)\right.$ $140 \mathrm{ps}(1.77 \%),\left(\tau_{2}\right) 500 \mathrm{ps}(95.43 \%)$, and $\left.\left(\tau_{3}\right) 1.87 \mathrm{~ns}(2.8 \%)\right)$. The observation confirms that the fluorescence quenching of CdS QDs in the presence of the dye is not due to electron transfer, but resonance energy transfer from donor QDs to acceptor OX1.

\section{Conclusion}

2-Mercaptoethanol capped CdS quantum dots (QDs) have been synthesized in aqueous solution. The QDs are characterized by using optical absorption/photoluminescence spectroscopy, powder X-ray diffraction (XRD), transmission electron microscopy (TEM), and picosecond resolved PL techniques and found to have average particle size of $4.0 \pm 0.5 \mathrm{~nm}$. However, in the aqueous solution the QDs are found to be aggregated to form nanostructure. Dynamic light scattering (DLS) experiments on the QDs in aqueous environments give an average particle size of $160 \mathrm{~nm}$, which is similar to those found in dried film by atomic force microscopy (AFM) $(150 \mathrm{~nm})$. TEM images of the aggregate also confirm a similar size of the nanoaggregate (150 $\mathrm{nm})$. The temperature-dependent DLS studies on the dialyzed aqueous solution of the nanoparticles indicate that the aggregation is essentially driven by noncovalent interactions between thiol-stabilized QDs.

Our studies demonstrate the nanoaggregate of the QDs to be an efficient host of small organic dye molecules of biological interest. A protein label, TNS, can easily be incorporated in the hydrophobic pores of the nanostructure. To estimate the average distance of a guest ligand molecule from a QD in the nanostructure we excited the QDs (donor) and followed Förster resonance energy transfer (FRET) dynamics to an organic dye, Oxazine 1 (acceptor), using steady state and time-resolved photoluminescence spectroscopy. The calculated energy transfer efficiency from CdS QDs (donor) to Oxazine 1 dye (acceptor) from steady state and picosecond-resolved studies is $74.3 \%$ and $77.64 \%$ respectively. The estimated donor-acceptor distances from steady state and time-resolved experiments are 26.5 and $25.7 \AA$ respectively. The donor-acceptor distance is consistent with the binding of the ligands at the surface site of the QDs. The techniques reported here could have implications on the future application of the QD-nanoaggregate as host of small substrate molecules of biological interest, which is one of the major research activities of this group.
Acknowledgment. S.S.N. thanks CSIR for a fellowship. We all thank DST for a financial grant (SR/FTP/PS-05/2004).

\section{References and Notes}

(1) Henglein, A. Chem. Rev. 1989, 89, 1861-1873.

(2) Alivisatos, A. P. J. Phys. Chem. 1996, 100, 13226-13239.

(3) Brus, L. E. J. Chem. Phys. 1984, 80, 4403-4409.

(4) Hu, K.; Brust, M.; Bard, A. J. Chem. Mater. 1998, 10, 11601165 . 620

(5) Dong, L.; Gushtyuk, T.; Jiao, J. J. Phys. Chem. B 2004, 108, 1617254.

(6) Yoshizawa, M.; Tamura, M.; Fujita, M. Science 2006, 312, 251-

(7) Douhal, A. Chem. Rev. 2004, 104, 1955-1976.

(8) Rao, K. V. S.; Srinivas, B.; Subrahmanyam, M. Photocatalytic reactions over semiconductor based zeolites for organic synthesis: A review; Research Signpost: Trivandrum, Kerala, India, 2004.

(9) Mahtab, R.; Rogers, J. P.; Singleton, C. P.; Murphy, C. J. J. Am. Chem. Soc. 1996, 118, 7028-7032.

(10) Lakowicz, J. R. Principles of fluorescence spectroscopy; Kluwer Academic/Plenum: New York, 1999.

(11) Fujii, H.; Inata, K.; Ohtaki, M.; Eguchi, K.; Arai, H. J. Mater. Sci. 2001, 36, 527-532.

(12) Rossetti, R.; Ellison, J. L.; Gibson, J. M.; Brus, L. E. J. Chem. Phys. 1984, 80, 4464-4469.

(13) Rossetti, R.; Nakahara, S.; Brus, L. E. J. Chem. Phys. 1983, 79, 1086-1088.

(14) Moffitt, M.; Eisenberg, A. Chem. Mater. 1995, 7, 1178-1184.

(15) He, R.; Qian, X.; Yin, J.; Xi, H.; Bian, L.; Zhu, Z. Colloids Surf. A 2003, 220, 151-157.

(16) Hasselbarth, A.; Eychmiiller, A.; Weller, H. Chem. Phys. Lett. 1993, 203, 271-276.

(17) Guinier, A. X-Ray diffraction in crystals, imperfect crystals and amorphous bodies; Dover Publication: New York, 1994.

(18) Cullity, B. D. Elements of X-ray Diffraction, 2nd ed.; AddisonWesley: London, 1978.

(19) Lakowicz, J. R.; Gryczynski, I.; Gryczynski, Z.; Nowaczyk, K.; Murphy, C. J. Anal. Biochem. 2000, 280, 128-136.

(20) Burda, C.; Chen, X.; Narayanan, R.; El-Sayed, M. A. Chem. Rev. 2005, 105, 1025-1102.

(21) Klimov, V. I. J. Phys. Chem. B 2000, 104, 6112-6123.

(22) Sarkar, R.; Shaw, A. K.; Narayanan, S. S.; Rothe, C.; Hintschich, S.; Monkman, A.; Pal, S. K. Opt. Mater. In press.

(23) Burda, C.; Link, S.; Mohamed, M.; El-Sayed, M. J. Phys. Chem. B 2001, 105, 12286-12292.

(24) McClure, W. O.; Edelman, G. M. Biochemistry 1966, 5, 19081919.

(25) Wang, R.; Bright, F. V. Appl. Spectrosc. 1993, 47, 792-799.

(26) Allan, J.; Hartman, P. G.; Crane-Robinson, C.; Aviles, F. X. Nature 1980, 288, 675-679.

(27) Cerf, C.; Lippens, G.; Muyldermans, S.; Segers, A.; Ramakrishnan, V.; Wodak, S. J.; Hallenga, K.; Wyns, L. Biochemistry 1993, 32, 1134511351

(28) Ramakrishnan, V. Curr. Opin. Struct. Biol. 1994, 4, 44-50.

(29) Zhong, D.; Pal, S. K.; Zewail, A. H. ChemPhysChem 2001, 2, 219227.

(30) Sarkar, N.; Das, K.; Das, S.; Datta, A.; Dutta, R.; Bhattacharyya, K. J. Chem. Soc., Faraday Trans. 1996, 92, 3097-3099.

(31) Sauer, M.; Drexhage, K. H.; Lieberwirth, U.; Müller, R.; Nord, S.; Zander, C. Chem. Phys. Lett. 1998, 284, 153-163.

(32) Efros, A. L. Phys. Rev. B 1992, 46, 7448.

(33) Petrov, E. P.; Cichos, F.; Zenkevich, E.; Starukhin, D.; Borczyskowski, C. v. Chem. Phys. Lett. 2005, 402, 233-238.

(34) Pal, S. K.; Mandal, D.; Sukul, D.; Bhattacharyya, K. Chem. Phys. 1999, 249, 63-71. 